\title{
Clinical Pharmacokinetics of Oral Semaglutide: Analyses of Data from Clinical Pharmacology Trials
}

\author{
Rune V. Overgaard ${ }^{1}$ (i) $\cdot$ Andrea Navarria ${ }^{2}$. Steen H. Ingwersen ${ }^{1} \cdot$ Tine A. Bækdal $^{1} \cdot$ Rasmus Juul Kildemoes $^{1}$ (i)
}

Accepted: 12 April 2021 / Published online: 10 May 2021

(C) The Author(s) 2021

\begin{abstract}
Objective The absorption, distribution and elimination of oral semaglutide, the first oral glucagon-like peptide-1 receptor agonist for treating type 2 diabetes, was investigated using a population pharmacokinetic model based on data from clinical pharmacology trials.

Methods A previously developed, two-compartment pharmacokinetic model, based on subcutaneous and intravenous semaglutide, was extended to include data from six oral semaglutide trials conducted in either healthy volunteers or subjects with renal or hepatic impairment. Five trials employed multiple doses of oral semaglutide (5-10 $\mathrm{mg}$ ) and one was a single-dose $(10 \mathrm{mg})$ trial. In a separate analysis, the model was re-estimated using data from a trial in subjects with type 2 diabetes.

Results The model accurately described concentration profiles across trials. Post-dose fasting time, co-ingestion of a large water volume, and body weight were the most important covariates affecting semaglutide exposure. Bioavailability was $0.8 \%$ when oral semaglutide was dosed using the recommended dosing conditions ( $30 \mathrm{~min}$ post-dose fasting time, administered with $\leq 120 \mathrm{~mL}$ of water), increasing with a longer post-dose fasting time and decreasing with higher water volume. Within-subject variability in bioavailability was $137 \%$, which with once-daily dosing and a long half-life translates into 33\% within-subject variability in steady-state exposure. There was no significant difference in oral bioavailability of semaglutide in healthy subjects and subjects with type 2 diabetes.
\end{abstract}

Conclusions The updated model provided a general characterisation of semaglutide pharmacokinetics following oral, subcutaneous and intravenous administration in healthy subjects and subjects with type 2 diabetes. Within-individual variation of oral bioavailability was relatively high, but reduced considerably at steady state.

ClinicalTrials.gov identifiers NCT01572753, NCT01619345, NCT02014259, NCT02016911, NCT02249871, NCT02172313, NCT02877355.

\section{Introduction}

Semaglutide is a long-acting glucagon-like peptide-1 (GLP-1) analogue approved for the treatment of type 2 diabetes (T2D) as a once-weekly subcutaneous (s.c.) formulation and, more recently, as a once-daily oral formulation [1]. Oral semaglutide is co-formulated with the absorption enhancer sodium $N$-(8-[2-hydroxybenzoyl]amino) caprylate, which facilitates the transcellular absorption of semaglutide across the gastric mucosa [2]. Significant reductions in glycated haemoglobin and body weight have been shown with oral semaglutide compared with placebo and several

Rune V. Overgaard

ruvo@novonordisk.com

Novo Nordisk A/S, Søborg, Denmark

Novo Nordisk A/S, Bagsvaerd, Denmark

\section{Key Points}

This population pharmacokinetic model for semaglutide provides a general characterisation of semaglutide pharmacokinetics across oral, subcutaneous and intravenous administration in healthy subjects and subjects with type 2 diabetes.

Dosing conditions (post-dose fasting time and water volume) of oral semaglutide influence semaglutide exposure and bioavailability.

The variability of exposure for oral semaglutide was accurately characterised, and demonstrated how the long half-life and daily dosing result in reduced variability at steady state. 
anti-diabetic agents [3-10]. Oral semaglutide has also been demonstrated to be well tolerated with a safety profile consistent with s.c. semaglutide and other GLP-1 receptor agonists [3-10]. Semaglutide is mainly distributed within the plasma volume, with a plasma protein binding of $>99 \%$ [11], and is extensively metabolised before excretion via urine and faeces [12].

Previously, a two-compartment pharmacokinetic (PK) model was used to describe semaglutide absorption, distribution and elimination, using data from nine clinical pharmacology trials, eight of which involved once-weekly s.c. administration and one involved once-weekly intravenous (i.v.) administration [13]. This s.c./i.v. model demonstrated that semaglutide PK were predictable, with low inter-individual and intra-individual variability across clinical pharmacology and phase III trials in healthy subjects and subjects with T2D [13, 14].

The objective of the present analysis was to extend the s.c./i.v. model to investigate the PK properties of orally administered semaglutide. Analyses included the effects of various dosing conditions (such as fasting time and water volume), demographic covariates and variability in exposure following single and multiple oral administration. Bioavailability in subjects with T2D was investigated in a separate analysis. This study provides a characterisation of the bioavailability for semaglutide, one of the first peptide-based treatments approved for oral administration, the first in diabetes, and the first with a large molecular weight $(4113.58 \mathrm{~g} / \mathrm{mol})$ [1].

\section{Materials and Methods}

\subsection{Clinical Data}

The model was developed using data from six clinical pharmacology trials that assessed: the effects of water volume and post-dose fasting (NCT01572753) [15], tablet degradation assessed by scintigraphy (NCT01619345) [16], renal impairment (NCT02014259) [17], hepatic impairment (NCT02016911) [18], drug-drug interaction with omeprazole (NCT02249871) [19] and food intake (NCT02172313) [20] on the PK of oral semaglutide (Table 1). These trials were selected because dosing of oral semaglutide was supervised on-site, which ensured adherence to the defined dosing conditions. The trials involved a range of dosing conditions, including the recommended instructions to take oral semaglutide in the fasting state in the morning, at least 30 min before the first food, beverage or other oral medications of the day and with no more than $120 \mathrm{~mL} / 4 \mathrm{fl} \mathrm{oz}$ of plain water [1]. In all trials, semaglutide $10 \mathrm{mg}$ was co-formulated with $300 \mathrm{mg}$ of sodium $N$-(8-[2-hydroxybenzoyl]amino) caprylate. The scintigraphy trial (NCT01619345) [16] involved a single dose of oral semaglutide $10 \mathrm{mg}$, whereas the other trials involved multiple dosing with 10 consecutive days of once-daily administration; doses were escalated after 5 days from 5 to $10 \mathrm{mg}$ in all multiple-dose trials except in the dosing conditions trial (NCT01572753) [15], in which $10 \mathrm{mg}$ was dosed for 10 days. Subjects included healthy volunteers and subjects with renal or hepatic impairment, a small number of whom also had T2D. Patients with endstage renal disease were excluded from this analysis.

Blood samples for PK assessment were obtained during the first 9 days of dosing (7-45 samples per subject) and following the last dose on day 10 (16-38 samples per subject). Plasma semaglutide concentrations were measured in plasma by a validated liquid chromatography-mass spectrometry assay following protein precipitation (Celerion Switzerland AG, Fehraltorf, Switzerland) using previously reported methods $[18,20]$ with a lower limit of quantification of $0.729 \mathrm{nmol} / \mathrm{L}$.

As the number of subjects with T2D in the main analysis was low $(N=16)$, to reliably estimate bioavailability in this population the model was re-estimated in a separate analysis. This additional analysis used data from a trial in subjects with T2D (NCT02877355) [21], which was not included in the main model because not all dosing was supervised. In this trial, subjects with T2D with $(N=36)$ or without $(N=19)$ upper gastrointestinal (GI) disease received oral semaglutide $3 \mathrm{mg}$ once daily for 5 days followed by $7 \mathrm{mg}$ for 5 days.

\subsection{Model Development Strategy}

The model was a modification of the previously developed two-compartment s.c./i.v. PK model for semaglutide [13]. To secure identifiability, the disposition model (including fixed-effects parameter values and variance of randomeffects parameters) was a fixed component in the current model. Oral semaglutide data were used to confirm the disposition model, and to estimate key aspects of oral absorption, including variability components and covariate factors. The oral absorption component was characterised by an oral bioavailability $(F)$ relative to the i.v. data from the s.c./i.v. model and by a first-order oral absorption rate constant $\left(k_{a}\right)$ (Fig. 1).

\subsection{Model Development Process}

The base model for oral semaglutide PK included the twocompartment disposition model described in Sect. 2.2, and first-order oral absorption with intra-individual variability for $k_{\mathrm{a}}$ and $F$, and additional inter-occasion variability for $F$ for each dose occasion. One-compartment and two-compartment models were investigated, confirming the suitability of a two-compartment disposition structure (Delta OFV, 


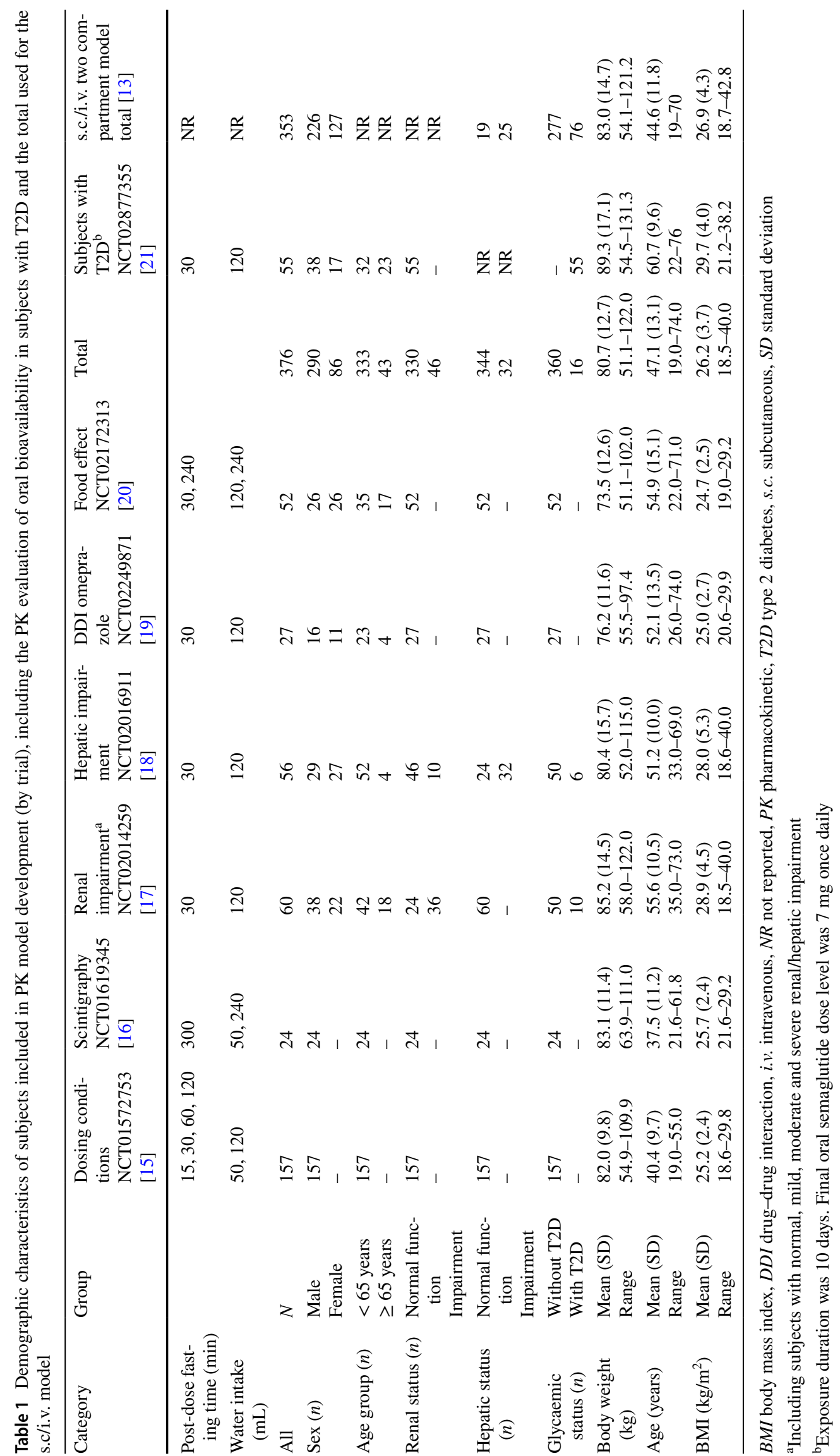


approximately 8000 points). The log-normal intra-individual variability/inter-occasion variability models were seen to adequately describe the estimated individual-occasion and between-occasion parameters, including $F$. The upper percentiles in estimated $F$ were far from $100 \%$; therefore, the log-normal distribution was adopted.

Covariates for the updated model for s.c./i.v./oral administration included the known covariate relationships for the s.c./i.v. disposition model, which were effects of body weight on clearance (CL), inter-compartment $\mathrm{CL}(Q)$, central volume $\left(V_{\mathrm{c}}\right)$ and peripheral volume $\left(V_{\mathrm{p}}\right)$, and effect of glycaemic status on CL. Additionally, the updated model included covariates on oral absorption parameters estimated in the current analysis, which were: body weight (continuous), post-dose fasting time $(15,30,60,120,240,300 \mathrm{~min})$ and water volume $(50,120,240 \mathrm{~mL})$ for $F$, and post-dose fasting time for $k_{\mathrm{a}}$. The relationship between post-dose fasting time and bioavailability (and absorption rate) was an exponential decay towards an asymptotic value, providing a flexible model that allows consistency with an absorption that stops at the time of food intake.

\subsection{Final Model}

Covariates retained in the model were identified by backwards elimination (criteria: change in objective function values $[\Delta \mathrm{OFV}>6.63$, corresponding to $p<0.01]$ ) to obtain a model with only significant covariates. Hepatic impairment $(\Delta \mathrm{OFV}=1.281)$, water volume $50 \mathrm{~mL}$ vs $120 \mathrm{~mL}(\Delta \mathrm{OFV}$ $=3.328)$, age $\geq 65$ years $(\Delta \mathrm{OFV}=3.024)$, $\operatorname{sex}(\Delta \mathrm{OFV}=$ $0.102)$ and renal impairment $(\Delta \mathrm{OFV}=2.099)$ were excluded in the final model (in that order). A trial factor was investigated for the single-dose scintigraphy trial, owing to a slight

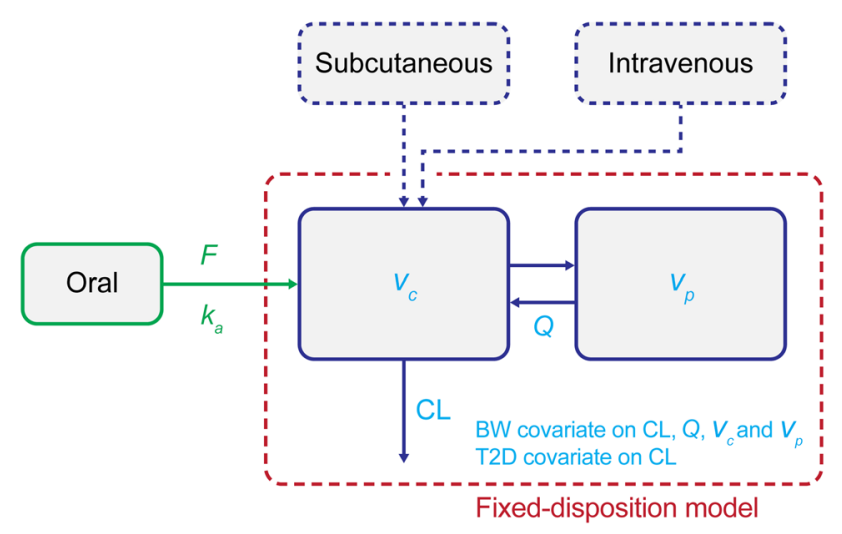

Fig. 1 Population pharmacokinetic model. Dashed boxes represent model components included in the previously developed subcutaneous/intravenous model. $B W$ body weight, $C L$ clearance, $F$ oral bioavailability, $k_{a}$ oral absorption rate constant, $Q$ inter-compartmental clearance, $T 2 D$ type 2 diabetes, $V_{c}$ central volume, $V_{p}$ peripheral volume under-estimation of concentrations in this trial by the model, but the trial factor was not statistically significant and was not included $(\triangle \mathrm{OFV}=5.749)$.

The final covariate models for subject $(i)$ and dosing occasion $(j)$ were parameterised as follows:

$k_{a i}=\left[\left(k_{a, \text { ref }}-\theta_{\text {ka.long.fast }}\right) \cdot \mathrm{e}^{-\theta_{T \text { fast }, k a} \cdot\left(T_{\text {fasting }}-30\right)}+\theta_{\text {ka.long.fast }}\right] \bullet \exp \left(\eta_{k a . i}\right)$

$C L_{i}=C L_{r e f} \cdot E_{\text {weight }, C L} \bullet E_{T 2 D} \cdot \exp \left(\eta_{C L . i}\right)$

$Q_{i}=Q_{r e f} \cdot E_{\text {weight }, C L} \cdot \exp \left(\eta_{C L . i}\right)$

$V_{c, i}=V_{c, r e f} \bullet E_{w e i g h t, V} \bullet \exp \left(\eta_{V c . i}\right)$

$V_{p, i}=V_{p, r e f} \bullet E_{\text {weight }, V} \bullet \exp \left(\eta_{V c . i}\right)$

$F_{i j}=F_{\text {ref }} \bullet E_{\text {weight }, F} \bullet E_{\text {water } . F} \bullet E_{\text {fasting }, F} \bullet \exp \left(\eta_{F . i}+\eta_{F . j}\right)$

where

$E_{\text {weight }, C L}=\left(\frac{\text { weight }_{i}}{85 \mathrm{~kg}}\right)^{\theta_{\text {weight }, C L}}$

$E_{\text {weight }, V}=\left(\frac{\text { weight }_{i}}{85 \mathrm{~kg}}\right)^{\theta_{\text {weight }, V}}$

$E_{T 2 D}=\left(\theta_{T 2 D, C L}\right)^{T 2 D}$

$E_{\text {weight }, F}=\left(\frac{\text { weight }_{i}}{85 \mathrm{~kg}}\right)^{\theta_{\text {weight }, F}}$

$E_{\text {water }, F}=\left(\theta_{240 m L}\right)^{240 m L}$

$E_{\text {fasting, } F}=\frac{1-e^{-\theta_{T, f a s t i n g, F} \cdot T_{\text {fasting }}}}{1-\mathrm{e}^{-\theta_{T, f a s t i n g, F} \cdot 30}}$

In the above equations, $\theta$ is the estimated (or fixed) value for each covariate, $\theta_{\text {ka.long.fast }}$ is the absorption rate constant for fasting times of $120 \mathrm{~min}$ or longer, $\eta_{i}$ is the subject-level random effects, $\eta_{j}$ is the occasion-level random effects; other exponents are indicator variables assigned the value 1 for the specific covariate values and 0 otherwise, $\mathrm{CL}_{\text {ref }}, Q_{\text {ref }}, V_{c, \text { ref }}$, $V_{p \text {,ref }}, F_{\text {ref }}$ and $k_{a \text {, ref }}$ are the parameter values for the reference subject profile $T_{\text {fasting }}$ post-dose fasting time ( $\mathrm{min}$ ).

The between-individual variance-covariance structure for $\mathrm{CL}$ and $V_{\mathrm{c}}$ was fixed to that of the s.c./i.v. disposition model. For $V_{\mathrm{p}}$, the between-individual variance was assigned the same value as for $V_{\mathrm{c}}$ and likewise, for $Q$ it was assigned the 
same value as for CL. Between-individual variations were estimated for $k_{\mathrm{a}}$ and for $F$. In addition, within-individual variability (i.e., variation between doses) was estimated for $F$. The residual error model was an additive error model for log-transformed concentrations.

\subsection{Model Predictions}

Because of substantial log-normal within-individual variability, standard computation of reference subject simulations, as well as population predictions and weighted residuals reported by the nonlinear mixed-effects modelling software (NONMEM) are poor measures to reflect data. Model fit and diagnostics were therefore provided based on full parametric simulations, which have been used in all figures.

\subsection{Software}

Datasets containing PK and covariate data from each trial were converted to a format compatible with the modelling software NONMEM (ICON Development Solutions, Ellicott City, MD, USA), Version 7.3. The software package R (Version 3.2.3, obtained from the R Foundation: http://www.rproject.org/foundation) was used for data file processing and analysis. In addition, PsN Version 4.60 (University of Uppsala, https://uupharmacometrics.github.io/PsN/index.html) was used as the interface for bootstrapping, search for global minima and creation of visual predictive checks.

\section{Results}

\subsection{Population Characteristics}

A total of 376 subjects with 14,897 observations from PK profiles were included in the PK model analysis. Demographic characteristics of subjects are shown in Table 1.

\subsection{Final Population PK Model Qualification and Characterisation of Semaglutide Pharmacokinetics Following Oral Administration}

The s.c./i.v. model [13] was updated to provide a general model also applicable to oral semaglutide. Details regarding parameter values, including inter-individual, inter-occasion and residual variability, are provided in Table 1 of the Electronic Supplementary Material (ESM). The model was qualified based on standard goodness-of-fit plots of predictions vs observed concentrations and weighted residuals, distribution of individual parameter estimates compared to s.c. modelling, visual predictive checks over each trial and across trials, mean model predictions across trial arms and individual fits over time. Qualification elements included the semaglutide concentration-time profiles, covariates, dosing conditions, consistency between oral and s.c./i.v. administration, and individual bioavailability estimates.

\subsubsection{Semaglutide Concentration-Time Profiles}

Without inclusion of trial factors, the model was able to accurately fit concentration levels across each of the 10-day trials, and thereby demonstrated consistency in PK for the six clinical pharmacology trials used for model development.

Diagnostic plots for the model showed that the model fits were accurate, and there were no critical trends in the conditional weighted residuals vs semaglutide concentration or time (Fig. 1 of the ESM). Focusing on the variability, the individual PK parameter values appeared to follow approximate log-normal distributions (Figs. 2 and 3 of the ESM). Additionally, the visual predictive checks showed that the model was able to accurately capture the median trends as well as the variability in semaglutide PK data for each individual trial (Fig. 4 of the ESM). The model demonstrated high accuracy for prediction of the median semaglutide profile and the $90 \%$ range.

Figure 2 presents the observed overall mean semaglutide concentrations and variability $(90 \%$ concentration ranges for within and between individuals) during and following 10 days of oral dosing at various dosing conditions. Following the final oral semaglutide dose at day 10, mean semaglutide concentration across subjects appeared to increase from a pre-dose value of $10 \mathrm{nmol} / \mathrm{L}$ to a maximum of $15 \mathrm{nmol} / \mathrm{L}$, both accurately predicted by the mean profile within $1 \mathrm{nmol} / \mathrm{L}$, despite the large variability (Fig. 2). Semaglutide plasma concentrations obtained from the single-dose scintigraphy trial (NCT01619345) [16] were slightly underestimated by the model (Fig. 3; and Fig. 4 of the ESM).

For simulation of typical profiles at steady state, mean parameter values are shown in Table 2 for the subset of the clinical pharmacology population who used the recommended dosing conditions for oral semaglutide $(N=209$ healthy subjects, $N=55$ subjects with T2D). Based on simulations, the average concentration at day 10 was $66 \%$ of exposure at steady state, and the accumulation ratio was estimated to be 12.6 from day 1 to steady state (computed via simulations including inter-individual variability and inter-occasion variability).

\subsubsection{Covariates}

The model predictions were also in accordance with the mean semaglutide concentration profiles when stratified according to covariate factors (Fig. 3). The model accurately described the semaglutide PK profile across covariate categories for oral semaglutide data, without modifying the 
disposition model, that provided an accurate fit to covariate categories for s.c. semaglutide [13]. Model predictions fitted accurately with observed trough profiles for post-dose fasting time, water volume, renal impairment and hepatic impairment (Fig. 3). The model also fitted accurately across the remaining covariates of sex, age and body weight (data not shown). Semaglutide average concentration $\left(C_{\text {avg }}\right)$ increased with longer duration of post-dose fasting time and decreased with co-administration of $240 \mathrm{~mL}$ of water (see Sect. 3.2.5). Average concentration also decreased in subjects with higher body weight. No other covariates affected semaglutide exposure.

\subsubsection{Influence of Dosing Conditions on Oral Semaglutide Bioavailability}

The model was used to illustrate the influence of dosing conditions on the absorption process of oral semaglutide. Using recommended dosing conditions for oral semaglutide, mean bioavailability was estimated at approximately $0.8 \%$ (0.795\% [95\% confidence interval 0.736-0.864]) (Table 1
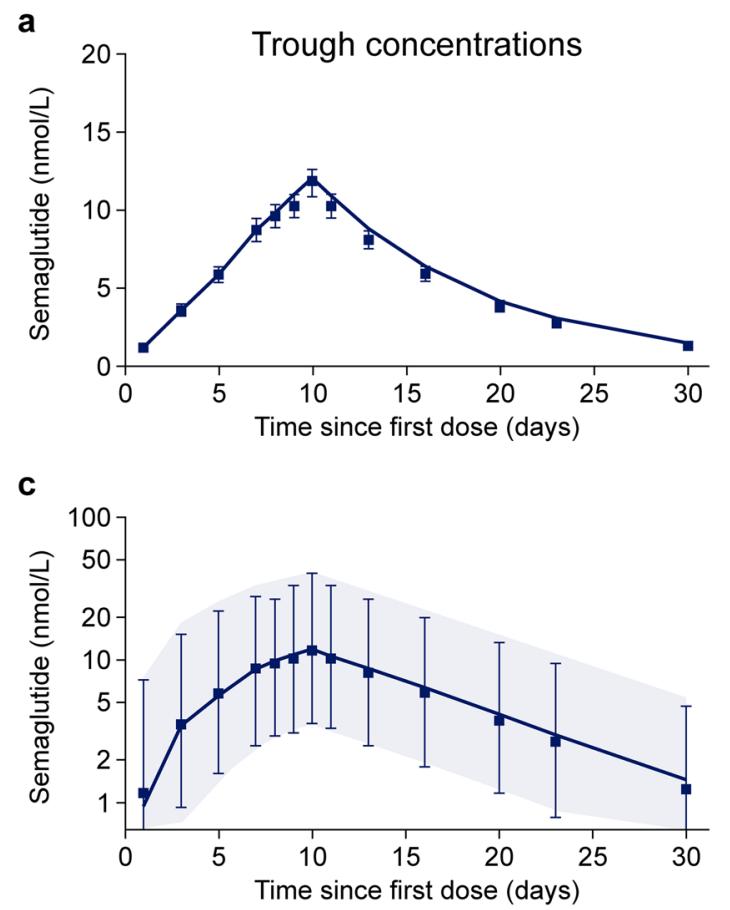

Fig. 2 Observed and model-predicted mean trough concentrations (with tail) across trials (a) and mean concentration profiles following last dose (b), both on an arithmetic scale; observed and modelpredicted geometric mean concentration profiles and 90\% range of trough concentrations since the first dose during 10 days of dosing (c) and following the last dose (d), both on a log scale. a, b Data points are observed geometric mean concentrations with $95 \%$ confidence intervals on an arithmetic scale. Lines are population predictions of the ESM). Bioavailability increased with longer postdose fasting time, reaching a plateau around $1.4 \%$ at 120 min (Fig. 4), and decreased with a higher water volume $(240 \mathrm{~mL})$. Bioavailability was independent of the volume of water between 50 and $120 \mathrm{~mL}$ (Fig. 4).

Simulated concentration profiles for deviations from the oral semaglutide recommended dosing conditions [1] are shown in Fig. 5. At steady state, single deviations such as a missed or double dose, a dose with shorter (15 $\mathrm{min})$ or longer (120 $\mathrm{min}$ ) post-dose fasting time or a dose taken with a larger volume of water $(240 \mathrm{~mL})$ will result in only minor and transient changes in exposure. However, persistent deviations from the recommended dosing conditions will lead to gradual changes in exposure over time.

\subsubsection{Consistency Between Semaglutide Pharmacokinetics for Oral and s.c./i.v. Administration}

To further qualify the similarities between the disposition models for oral, s.c. and i.v. dosing of semaglutide, estimated individual parameter values obtained from the oral model
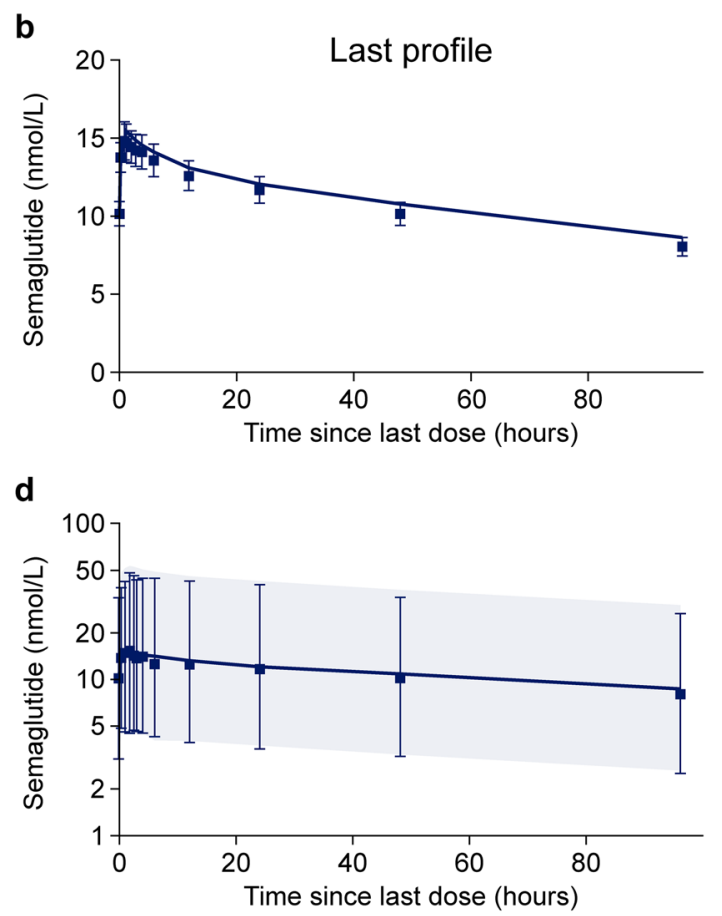

from the final model. c, d Data points are observed geometric mean concentrations with $90 \%$ ranges on a semi-logarithmic scale. Shaded areas are model-predicted geometric mean and $90 \%$ prediction intervals based on approximately 10,000 simulated profiles using the final population pharmacokinetic model. Data are from the following trials: dosing conditions (NCT01572753) [15], renal (NCT02014259) [17], hepatic (NCT02016911) [18], drug-drug interaction omeprazole (NCT02249871) [19] and food effect (NCT02172313) [20] 
Table 2 Mean estimated semaglutide PK parameters in healthy subjects and in subjects with T2D using the recommended dosing conditions for oral semaglutide

\begin{tabular}{lclllllll}
\hline Analysis & $N$ & $\begin{array}{l}\mathrm{CL} \\
(\mathrm{L} / \mathrm{h})\end{array}$ & $\begin{array}{l}V_{\mathrm{c}} \\
(\mathrm{L})\end{array}$ & $\begin{array}{l}V_{\mathrm{p}} \\
(\mathrm{L})\end{array}$ & $\begin{array}{l}V_{\text {total }} \\
(\mathrm{L})\end{array}$ & $\begin{array}{l}Q \\
(\mathrm{~L} / \mathrm{h})\end{array}$ & $\begin{array}{l}k_{\mathrm{a}} \\
\left(\mathrm{h}^{-1}\right)\end{array}$ & $\begin{array}{l}F \\
(\%)\end{array}$ \\
\hline Healthy subjects & 209 & 0.033 & 3.4 & 3.9 & 7.2 & 0.28 & 2.09 & 0.76 \\
Subjects with T2D & 55 & 0.039 & 3.7 & 4.2 & 7.9 & 0.31 & 2.09 & 0.69 \\
\hline
\end{tabular}

Data are geometric means of the typical PK parameter values for each subject. Data are from the following trials: healthy subjects: dosing conditions (NCT01572753) [15], scintigraphy (NCT01619345) [16], renal (NCT02014259) [17], hepatic (NCT02016911) [18], DDI omeprazole (NCT02249871) [19] and food effect (NCT02172313) [20]. Subjects with T2D: T2D with or without upper GI disease (NCT02877355) [21]

$C L$ clearance, $D D I$ drug-drug interaction, $F$ bioavailability, $G I$ gastrointestinal, $k_{a}$ absorption rate, $N$ number of subjects, $P K$ pharmacokinetic, $Q$ inter-compartmental clearance, $t_{1 / 2}$ plasma half-life, $T 2 D$ type 2 diabetes, $V_{c}$ central volume, $V_{p}$ peripheral volume, $V_{\text {total }}$ total volume

were compared to, and found to be in accordance with, the expected values from the s.c./i.v. model. A complete overlap of the individual estimated disposition $\mathrm{PK}$ parameter value $\left(\mathrm{CL}, V \mathrm{c}, Q, V_{\mathrm{p}}\right.$ ) distributions, and the expected distributions (based on demographic characteristics) of the parameter values obtained from the s.c./i.v. model was observed (Fig. 2 of the ESM). This indicates similar disposition models for oral, s.c. and i.v. dosing of semaglutide.

\subsubsection{Individual Bioavailability Estimates}

The model was used to obtain individual bioavailability estimates for each subject and each dose administration. The link between the observed individual PK profiles and the variability in bioavailability is illustrated in Fig. 3 of the ESM, for 12 subjects during and following ten oral semaglutide doses. Subjects were selected to include the highest, medium and lowest range of bioavailabilities. The figure indicates a fairly accurate assessment of individual parameters, as well as dose-to-dose differences in bioavailability. This qualifies the subsequent assessment of variability and the approximately log-normal distribution of bioavailability estimates observed across dose administrations (Fig. 3 of the ESM).

\subsubsection{Variability of Semaglutide Exposure and Bioavailability at Steady State}

The variability of exposure and bioavailability for oral semaglutide is illustrated in Fig. 6. Total variability of exposure (area under the curve) after a single dose of oral semaglutide was estimated to be $166 \%$, reduced to $68 \%$ at steady state. The within-subject variability of bioavailability (equivalent to the within-subject variability in exposure after a single dose) was estimated to be $137 \%$, whereas at steady state, the within-subject variability in exposure was reduced to $33 \%$. Between-individual variability was estimated to be $56 \%$ after a single dose as well as at steady state. Data from the dosing conditions trial (NCT01572753) [15] confirmed the above model-derived conclusions regarding bioavailability: with repeated dosing, the exposure variability was reduced, and the $90 \%$ exposure range reduced from approximately 20 -fold after the first dose to around five-fold after the tenth dose (Fig. 6 of the ESM).

\subsection{Exposures at Steady State}

Because ratios may differ between demographic subgroups at day 10 and at steady state (in particular, if a difference in half-life is present), the model was used to calculate the steady-state exposure difference. The model demonstrated that exposure ratios between the subgroups were similar or slightly smaller on day 10 compared with steady state (details not shown), thereby confirming that the primary conclusions regarding exposure ratios across the 10-day trials are applicable at steady state.

\subsection{Evaluation of Semaglutide Exposures and Bioavailability Following Oral Administration in Subjects with T2D With or Without Upper GI Disease}

The re-estimated model based on data from the trial in 55 subjects with T2D with or without upper GI disease [21] included covariate factors for T2D and for upper GI disease. Modelling equations and patient demographic characteristics are shown in the ESM and Table 1, respectively.

In accordance with the conclusions from the trial [21], the modelled PK profiles were similar for subjects with or without upper GI disease (Fig. 7 of the ESM), suggesting that the presence of upper GI disease does not affect semaglutide PK. Mean estimated parameter values in subjects with T2D dosed with oral semaglutide according to the recommended conditions are shown in Table 2. Total volume was $7.9 \mathrm{~L}$ and $\mathrm{CL}$ was $0.039 \mathrm{~L} / \mathrm{h}$ in subjects with T2D. The estimated diabetes status factor on bioavailability for subjects with T2D was 0.841 (95\% confidence interval 0.607-1.15); therefore, there was no significant difference between the oral bioavailability of semaglutide in subjects with T2D and healthy subjects. 


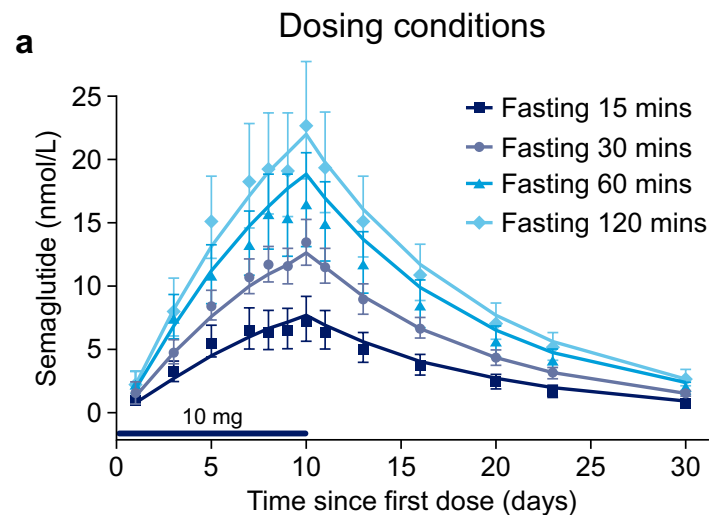

c
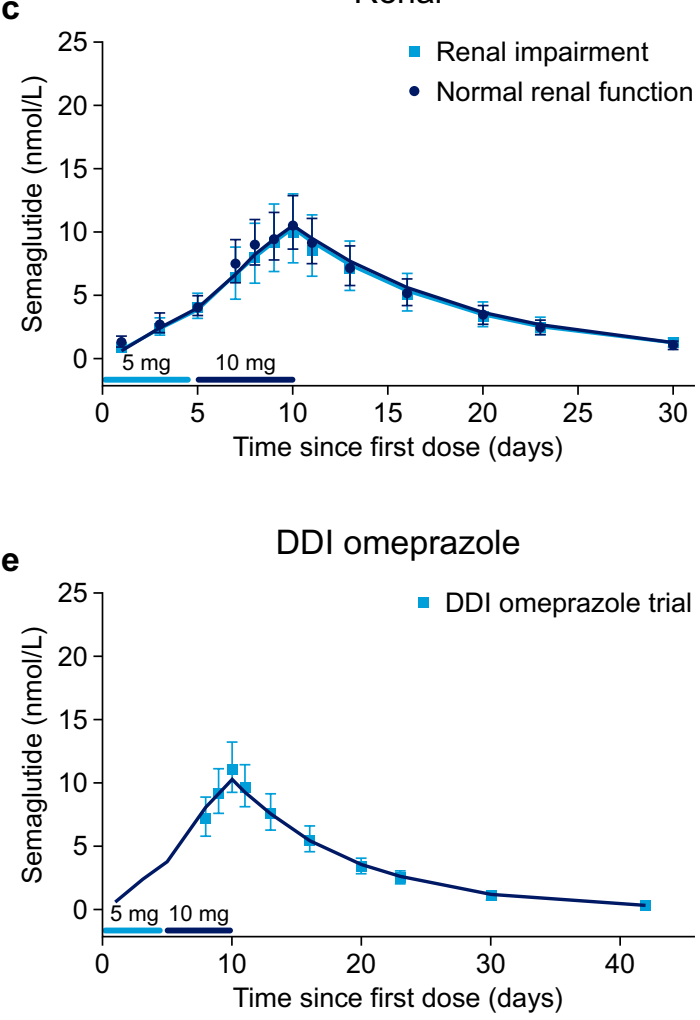

Fig. 3 Observed and model-predicted trough concentration-time profiles by trial and key covariates in each trial: post-dose fasting time in the dosing conditions trial (a), water volume in the scintigraphy trial (b), renal impairment in the renal trial (c), hepatic impairment in the hepatic trial (d) and during repetitive dosing (a, c, d) and following a single dose (b); observed concentrations and model-predicted full semaglutide pharmacokinetic profiles by trial for the drug-drug

\section{Discussion}

A population PK model of oral semaglutide was developed and validated in the current study and found suitable for accurately describing the PK properties across demographic

\section{b Scintigraphy}

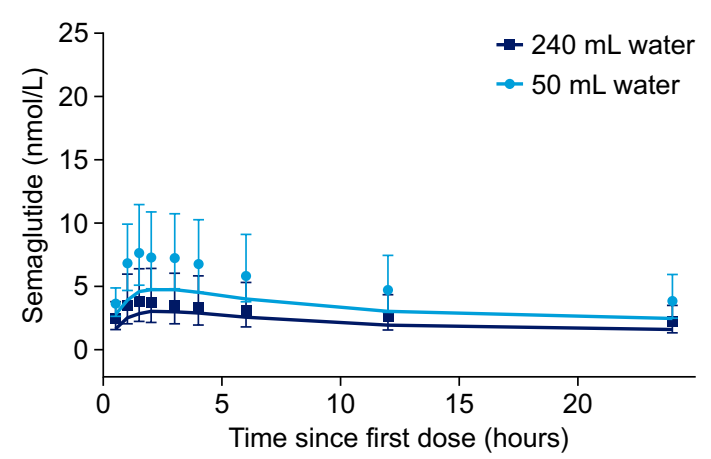

d
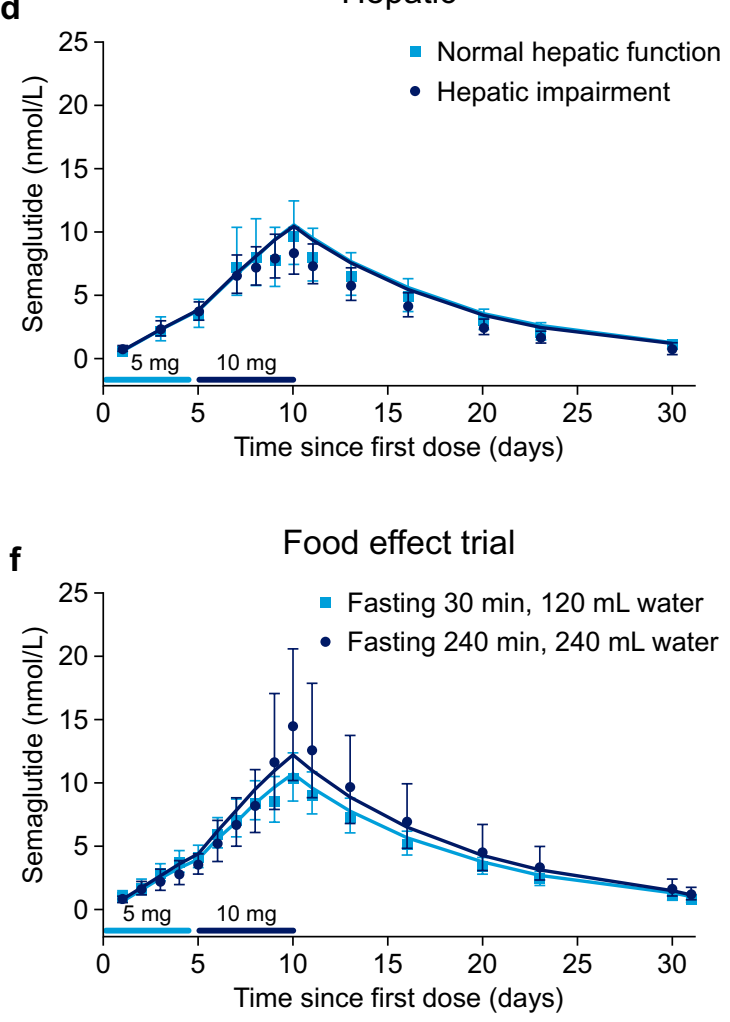

interaction (DDI) omeprazole and food-effect trials (e, f). Data points with error bars are observed geometric mean concentrations with $95 \%$ confidence intervals. Lines are population predictions. Data are from the following trials: dosing conditions (NCT01572753) [15], scintigraphy (NCT01619345) [16], renal (NCT02014259) [17], hepatic (NCT02016911) [18], DDI omeprazole (NCT02249871) [19] and food effect (NCT02172313) [20]

covariates and dosing conditions and for simulating PK profiles in subjects dosed with oral semaglutide.

Model-based analysis of semaglutide PK indicated that, once absorbed, semaglutide is distributed, metabolised and eliminated in the same way, irrespective of the route of administration. Distribution and elimination were similar 


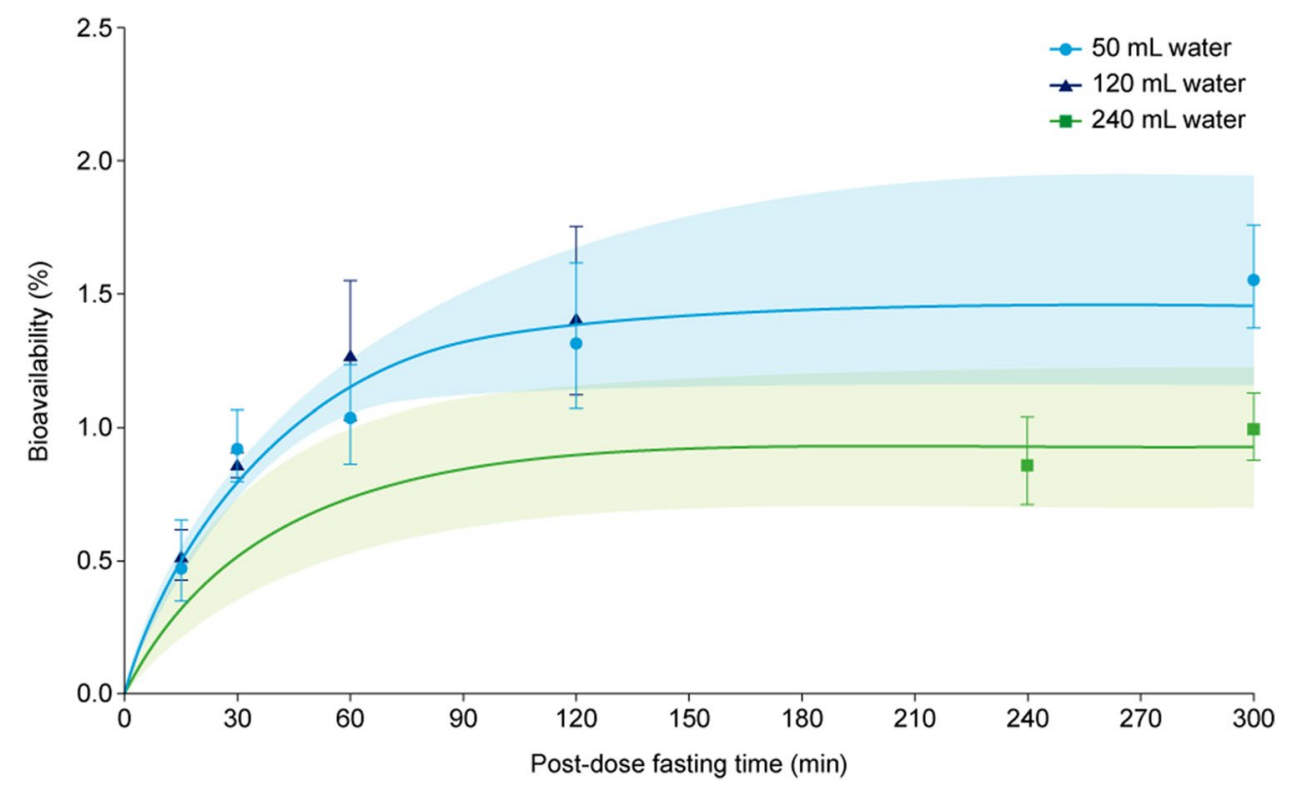

Fig. 4 Absolute bioavailability vs post-dose fasting time by water volume. Data points are means with $95 \%$ confidence intervals of individual model-derived parameter estimates. Lines are model-derived relationships to the fasting time and shaded areas are $95 \%$ confidence intervals obtained by bootstrapping. The model does not discriminate between 50 and $120 \mathrm{~mL}$, and therefore the

for oral, s.c. and i.v. semaglutide, and were characterised by two-compartment PK and first-order elimination. Furthermore, s.c. and oral administration are both consistent with a first-order absorption process, although oral absorption appears to stop soon after the time of meal ingestion. Compared with s.c. administration, oral absorption was significantly faster and bioavailability was lower (and therefore more variable) [22]. The day-to-day variability in oral absorption was reduced by frequent dosing and the long half-life of semaglutide, leading to stable steady-state concentrations for oral semaglutide.

Covariates for the model were implemented on multiple parameters, to reflect physiologically plausible mechanisms of covariate effects. Disposition parameter covariates were informed by s.c. and i.v. data, which are subject to much less variability, whereas covariates on oral absorption rate and bioavailability were informed by data obtained following oral administration. Body weight was the only intrinsic covariate that affected exposure; however, oral semaglutide provides adequate systemic exposure over the body weight range of 40-188 kg evaluated in clinical trials [4, 5, 8-10, 23]. Demographic covariates such as sex, age, and hepatic or renal impairment had no or a minor influence on the exposure of oral semaglutide. The exposure of semaglutide was, however, found to increase with a longer post-dose fasting time and decrease when oral semaglutide was coadministered with a water volume of $240 \mathrm{~mL}$, double that modelled lines are identical for these two water volumes. Data are from the following trials: dosing conditions (NCT01572753) [15], scintigraphy (NCT01619345) [16], renal (NCT02014259) [17], hepatic (NCT02016911) [18], drug-drug interaction omeprazole (NCT02249871) [19] and food effect (NCT02172313) [20]

of the recommended dosing conditions [1]. The impact of covariates on the PK of semaglutide in the current analysis supports previous findings for s.c. semaglutide, that no dose adjustment is needed across population subgroups [13, 14, 24]. This consistency of covariate results from the current oral semaglutide study with those from s.c. dosing of semaglutide indicates that the pharmacodynamic effects of oral semaglutide are independent of the route of administration.

Being a large peptide molecule and hence difficult to absorb, the bioavailability of semaglutide dosed orally is relatively low $[25,26]$. When using the recommended dosing conditions for oral semaglutide (30 min fasting and up to $120 \mathrm{~mL}$ of water) [1], the bioavailability was $0.8 \%$. Alteration of the recommended dosing conditions resulted in increased oral semaglutide bioavailability with a longer post-dose fasting time and decreased bioavailability with coadministration of a greater water volume of $240 \mathrm{~mL}$. Bioavailability was independent of water volumes up to $120 \mathrm{~mL}$. These results are consistent with the known mechanisms of oral semaglutide absorption, as demonstrated by human in vivo studies with radioactively labelled tablets [2]. These studies showed that the absorption of semaglutide takes place in the stomach and is sensitive towards other constituents in the gastric fluid. Hence, oral semaglutide absorption is impaired by the presence of food and larger amounts of water in the stomach [2]. Model-simulated concentration profiles demonstrated that at steady state, single deviations 

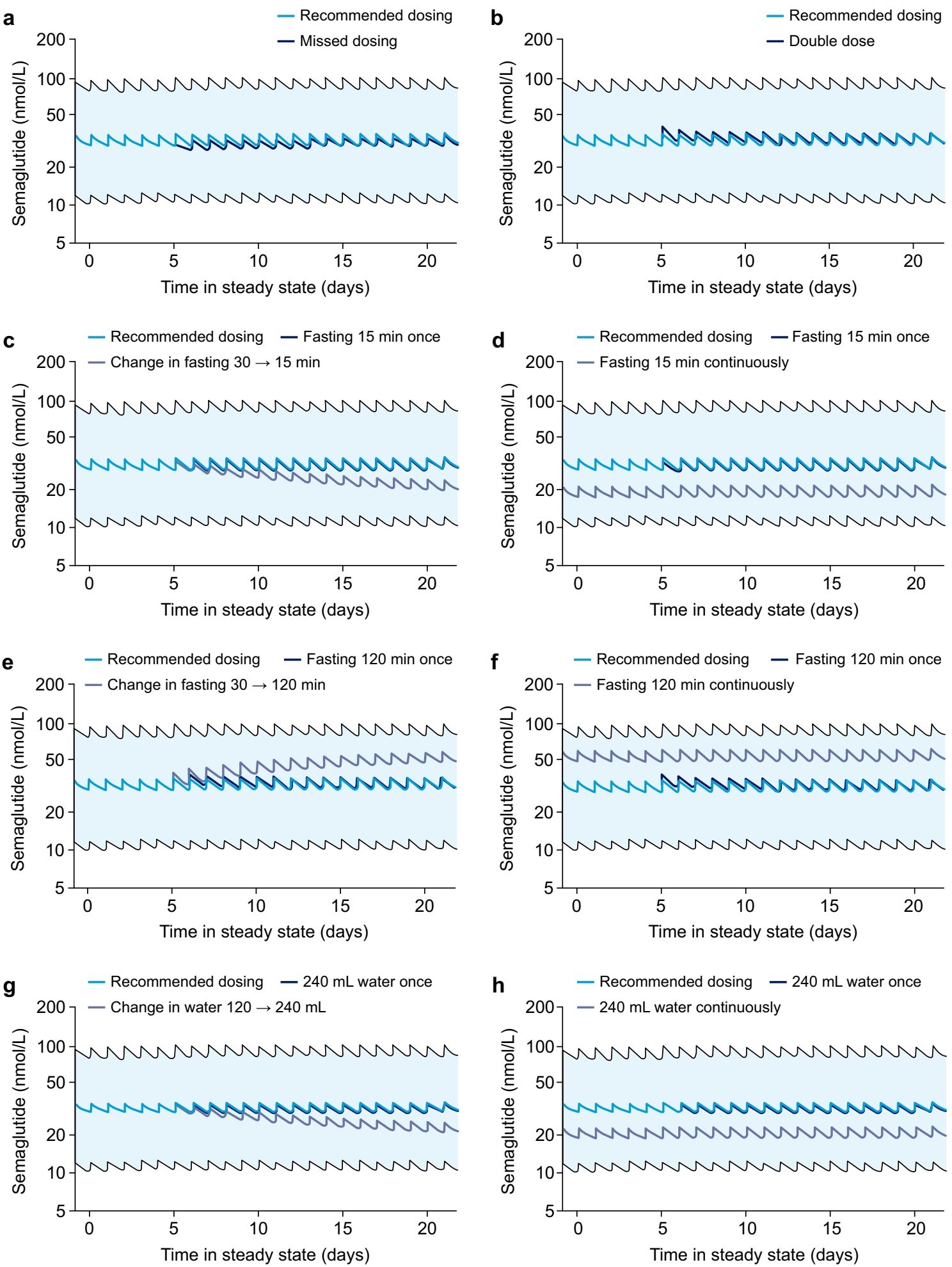

Fig. 5 Concentration profiles for deviations from the recommended dosing regimen and dosing conditions for once-daily dosing of $14 \mathrm{mg}$ of semaglutide: one missed dose day 5 (a); two doses day 5 (b); change of post-dose fasting to $15 \mathrm{~min}$ from day 5 (c); change of post-dose fasting to $15 \mathrm{~min}$ throughout (d); change of post-dose fasting to $120 \mathrm{~min}$ from day 5 (e); change of post-dose fasting to 120 min throughout (f); change to administration with $240 \mathrm{~mL}$ of water

from day 5 (g); and change to administration with $240 \mathrm{~mL}$ of water throughout $(\mathbf{h})$. Data are population predictions with $90 \%$ prediction intervals. Illustrated are recommended dosing conditions (dark blue lines) and deviations from the dosing regimen and dosing conditions (light blue and purple lines). Recommended dosing conditions: administration in a fasting state with up to $120 \mathrm{~mL}$ of water and postdose fasting time of at least $30 \mathrm{~min}$ 

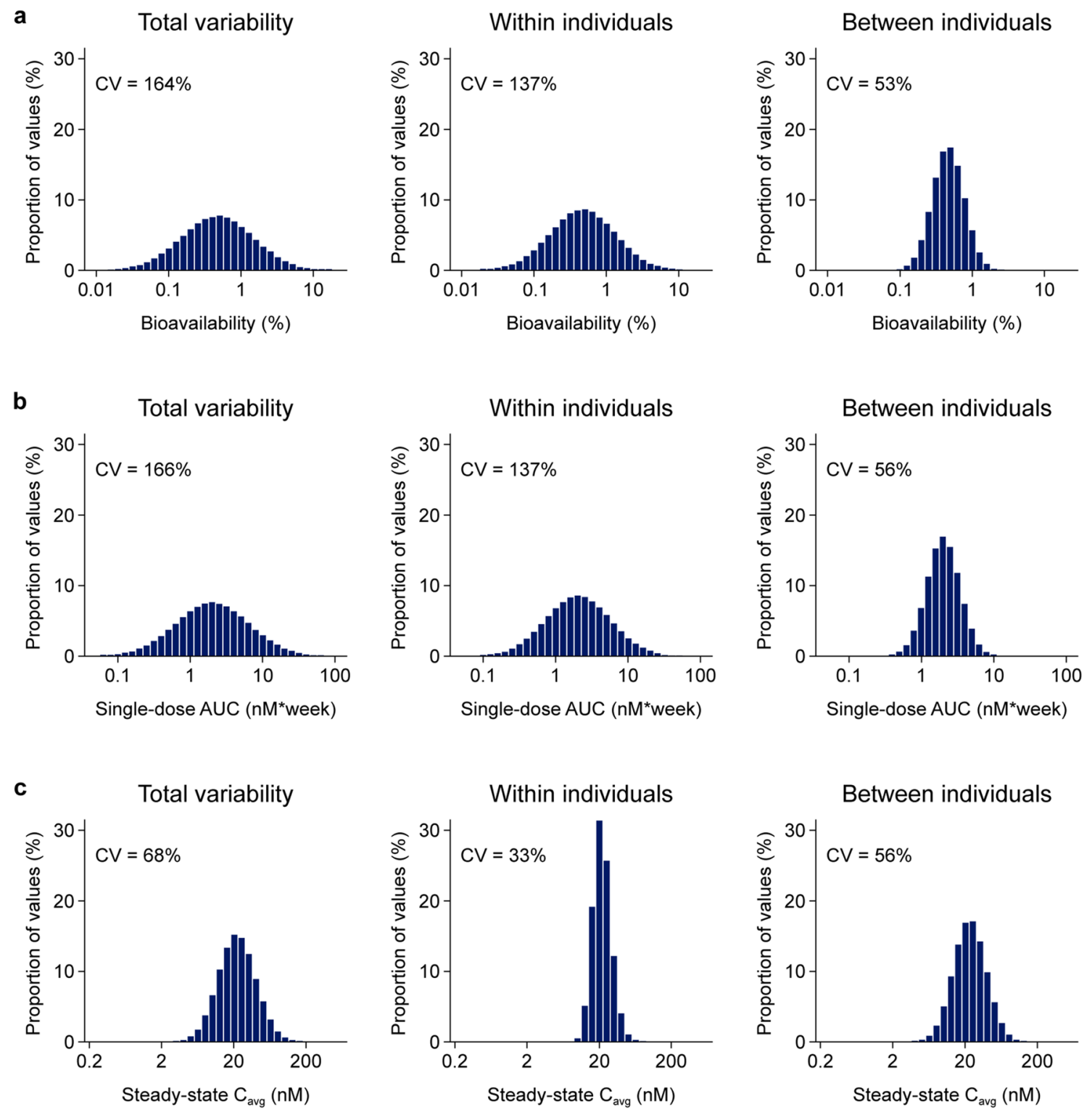

Fig. 6 Distribution of variability components for the oral bioavailability of semaglutide (a), area under the curve (AUC) values following a single oral dose (b), and average concentration $\left(C_{\text {avg }}\right)$ at steady-state dosing of $10 \mathrm{mg}$ of semaglutide once daily (c). Data are

from the oral semaglutide recommended dosing conditions have a minimal impact on exposure.

The model was used to evaluate the consistency of exposures in the clinical pharmacology trials used for model development and identify the fundamental PK properties for oral semaglutide. There was general consistency across trials, and the model qualified that primary analyses obtained at day 10 are expected to be valid at steady state.

Considerable between-individual and within-individual variability was observed for oral semaglutide bioavailability, which is usual for molecules with low bioavailability [22]. The once-daily dosing frequency combined with the long model-derived bioavailabilities and exposures based on simulations of a reference subject profile using the final population pharmacokinetic model. $C V$ coefficient of variation

plasma half-life of semaglutide ( 1 week) cause multiple previous doses to contribute to the exposure at steady state; the most recent dose will on average contribute approximately $10 \%$ of the total exposure. Therefore, at steady state, the variability in total and within-subject variability in exposure is reduced compared with after a single dose. In contrast, this has no impact on the between-subject variability, which was similar both after single-dose oral semaglutide and at steady state.

In a separate analysis involving re-estimation of the model using data from a trial in subjects with T2D with or without upper GI disease [21], oral semaglutide PK were found to 
be similar between healthy subjects and subjects with T2D with or without upper GI disease. No significant differences in oral bioavailability were identified between subjects with T2D and healthy subjects, which was in accordance with the results from the trial reported by Granhall and colleagues [27], which demonstrated similar semaglutide exposure in male subjects with and without T2D.

During planning of the original analysis, a stepwise approach was judged to be superior to a joint estimation of all data (s.c., i.v. and oral semaglutide). The disposition model was robustly estimated based on s.c. and i.v. data alone, with a low relative standard error (between 3 and 9\%) and low variability [13]. As clinical pharmacology data of oral semaglutide were characterised by high within-subject variability, potential changes to the disposition model obtained by including oral data were judged to be less credible, as the changes could be the result of changed estimation procedures and difficulties with the estimation. Nevertheless, a sensitivity analysis was conducted with a joint estimate of oral, s.c. and i.v. data, providing similar results as the original analysis.

Whereas the presented analysis provides a general description of semaglutide PK, there are a few limitations of potential relevance. The i.v. data in the original s.c./i.v. model [13] were from a single trial, leading to some uncertainty in the fixed disposition parameters. However, the uncertainty in the s.c./i.v. model is very low, with a low relative standard error for the disposition parameter estimates [13]. In the current study, the distribution of bioavailabilities was appropriately described with a log-normal distribution and the upper percentiles were substantially lower than $100 \%$, despite the log-normal distribution theoretically having no upper end. It should be noted, however, that it is not realistic to use this model to simulate extreme individual cases.

Our analysis did not investigate other factors such as alcohol, smoking, different disease characteristics or many potential concomitant glucose-lowering medications. The impact of reducing gastric $\mathrm{pH}$ with omeprazole on the $\mathrm{PK}$ of oral semaglutide has been assessed and this may cause a small but not clinically relevant increase in semaglutide exposure [19]. Administration of placebo tablets concomitantly with oral semaglutide can reduce exposure to semaglutide by $35 \%$ in healthy subjects [28] and reinforces the dosing conditions to avoid administering other oral medications within 30 minutes of oral semaglutide $[1,28]$.

The single-dose trial appeared to have slightly higher exposure compared with the other trials in the dataset (although this was not statistically significant at the $p=0.01$ level), indicating that a trial factor could be present. The scintigraphy study had a rigid design not representative of real life, and the model underestimated the scintigraphy trial data.
In general, estimated parameters were robust across a number of sensitivity analyses, including estimates of bioavailability and the associated inter-individual and withinindividual variation. These estimates reflect a clinical trial setting in which dose and administration are supervised, so therefore may not provide an accurate estimate of absorption variability and may differ from a real-world T2D setting. Likewise, steady-state exposure levels can be derived from the developed model, both for healthy subjects and subjects with T2D. Pharmacokinetic data derived from phase III trials are expected to provide a more accurate real-world indication of exposure levels when oral semaglutide is administered for a longer period outside of the controlled clinical trial setting.

\section{Conclusions}

This updated population PK model provided a general characterisation of semaglutide PK across oral, s.c. and i.v. administration, demonstrating similar PK for healthy subjects and subjects with T2D. Bioavailability and exposure of oral semaglutide were reduced by shorter post-dose fasting time (15 min) and co-administration of a larger water volume $(240 \mathrm{~mL})$. The within-individual variation from dose to dose in oral semaglutide bioavailability was relatively high, but as a result of daily dosing and a long plasma half-life (1 week), the variability of semaglutide exposure at steady state was considerably reduced.

Supplementary Information The online version contains supplementary material available at https://doi.org/10.1007/s40262-021-01025-x.

Acknowledgements The authors thank the patients, investigators, trial-site staff and Novo Nordisk employees involved in the trials. In addition, the authors thank Lindsay Uglow of Spirit Medical Communications Group Limited for medical writing and editorial assistance (funded by Novo Nordisk A/S) and Christin L $\varnothing$ th Hertz of Novo Nordisk for reviewing the manuscript.

\section{Declarations}

Funding This study was sponsored by Novo Nordisk A/S.

Conflicts of Interest/Competing Interests Rune V. Overgaard, Andrea Navarria, Steen H. Ingwersen, Tine A. Bækdal and Rasmus Juul Kildemoes are employees of, and shareholders in, Novo Nordisk A/S.

Ethics Approval This article is based on previously conducted studies and does not contain any studies with human participants or animals performed by any of the authors.

Consent to Participate Not applicable.

Consent for Publication Not applicable. 
Code Availability Available upon reasonable request. See Availability of Data and Material section.

Availability of Data and Material The model code and data sets will be shared with researchers submitting a research proposal approved by the independent review board. Access request proposals can be found at www.novonordisk-trials.com. Data will be made available after research completion, and approval of the product and product use in the European Union and the USA. Individual participant data will be shared in data sets in a de-identified and anonymised format, with no limitations on how the data can be used.

Authors' Contributions All authors contributed to the study conception and design. Material preparation, data collection and analysis were performed by RVO and RJK. The first draft was developed by RVO and RJK with the help of medical writing support. All authors commented on all versions of the manuscript and approved the final version.

Open Access This article is licensed under a Creative Commons Attribution-NonCommercial 4.0 International License, which permits any non-commercial use, sharing, adaptation, distribution and reproduction in any medium or format, as long as you give appropriate credit to the original author(s) and the source, provide a link to the Creative Commons licence, and indicate if changes were made. The images or other third party material in this article are included in the article's Creative Commons licence, unless indicated otherwise in a credit line to the material. If material is not included in the article's Creative Commons licence and your intended use is not permitted by statutory regulation or exceeds the permitted use, you will need to obtain permission directly from the copyright holder. To view a copy of this licence, visit http://creativecommons.org/licenses/by-nc/4.0/.

\section{References}

1. US Food and Drug Administration. Rybelsus ${ }^{\circledR}$ : prescribing information. https://www.accessdata.fda.gov/drugsatfda_docs/label/ 2020/213182s000,213051s001lbl.pdf. Accessed 11 Nov 2020.

2. Buckley ST, Bækdal TA, Vegge A, Maarbjerg SJ, Pyke C, Ahnfelt-Rønne J, et al. Transcellular stomach absorption of a derivatized glucagon-like peptide-1 receptor agonist. Sci Transl Med. 2018;10(467):eaar7047.

3. Davies M, Pieber TR, Hartoft-Nielsen ML, Hansen OKH, Jabbour S, Rosenstock J. Effect of oral semaglutide compared with placebo and subcutaneous semaglutide on glycemic control in patients with type 2 diabetes: a randomized clinical trial. JAMA. 2017;318(15):1460-70.

4. Aroda VR, Rosenstock J, Terauchi Y, Altuntas Y, Lalic NM, Morales Villegas EC, et al. PIONEER 1: randomized clinical trial of the efficacy and safety of oral semaglutide monotherapy in comparison with placebo in patients with type 2 diabetes. Diabetes Care. 2019;42:1724-32.

5. Mosenzon O, Blicher TM, Rosenlund S, Eriksson JW, Heller S, $\mathrm{Hels} \mathrm{OH}$, et al. Efficacy and safety of oral semaglutide in patients with type 2 diabetes and moderate renal impairment (PIONEER 5): a placebo-controlled, randomised, phase 3 a trial. Lancet Diabetes Endocrinol. 2019;7:515-27.

6. Pieber TR, Bode B, Mertens A, Cho YM, Christiansen E, Hertz $\mathrm{CL}$, et al. Efficacy and safety of oral semaglutide with flexible dose adjustment versus sitagliptin in type 2 diabetes (PIONEER 7): a multicentre, open-label, randomised, phase 3a trial. Lancet Diabetes Endocrinol. 2019;7:528-39.

7. Pratley R, Amod A, Hoff ST, Kadowaki T, Lingvay I, Nauck $\mathrm{M}$, et al. Oral semaglutide versus subcutaneous liraglutide and placebo in type 2 diabetes (PIONEER 4): a randomised, doubleblind, phase 3a trial. Lancet. 2019;394:39-50.

8. Rodbard HW, Rosenstock J, Canani LH, Deerochanawong C, Gumprecht J, Lindberg $S \varnothing$, et al. Oral semaglutide versus empagliflozin in patients with type 2 diabetes uncontrolled on metformin: the PIONEER 2 Trial. Diabetes Care. 2019;42:2272-81.

9. Rosenstock J, Allison D, Birkenfeld AL, Blicher TM, Deenadayalan S, Jacobsen JB, et al. Effect of additional oral semaglutide vs sitagliptin on glycated hemoglobin in adults with type 2 diabetes uncontrolled with metformin alone or with sulfonylurea: the PIONEER 3 randomized clinical trial. JAMA. 2019;321:1466-80.

10. Zinman B, Aroda VR, Buse JB, Cariou B, Harris SB, Hoff ST, et al. Efficacy, safety, and tolerability of oral semaglutide versus placebo added to insulin with or without metformin in patients with type 2 diabetes: the PIONEER 8 trial. Diabetes Care. 2019;42:2262-71.

11. Jensen L, Kupcova V, Arold G, Pettersson J, Hjerpsted JB. Pharmacokinetics and tolerability of semaglutide in people with hepatic impairment. Diabetes Obes Metab. 2018;20:998-1005.

12. Jensen L, Helleberg H, Roffel A, van Lier JJ, Bjornsdottir I, Pedersen PJ, et al. Absorption, metabolism and excretion of the GLP-1 analogue semaglutide in humans and nonclinical species. Eur J Pharm Sci. 2017;104:31-41.

13. Overgaard RV, Delff PH, Petri KCC, Anderson TW, Flint A, Ingwersen SH. Population pharmacokinetics of semaglutide for type 2 diabetes. Diabetes Ther. 2019;10:649-62.

14. Petri KCC, Ingwersen SH, Flint A, Zacho J, Overgaard RV. Semaglutide s.c. once-weekly in type 2 diabetes: a population pharmacokinetic analysis. Diabetes Ther. 2018;9:1533-47.

15. Donsmark M, Borregaard J, Breitschaft A, Bækdal TA, Søndergaard FL. Evaluation of the effects of water volume with dosing and post-dose fasting period on pharmacokinetics of oral semaglutide. Diabetologia. 2017;60(Suppl 1):S363-4.

16. Connor A, Donsmark M, Hartoft-Nielsen M-L, Søndergaard FL, Bækdal TA. A pharmacoscintigraphic study of the relationship between tablet erosion and pharmacokinetics of oral semaglutide. Diabetologia. 2017;60(Suppl 1):S361.

17. Granhall C, Sondergaard FL, Thomsen M, Anderson TW. Pharmacokinetics, safety and tolerability of oral semaglutide in subjects with renal impairment. Clin Pharmacokinet. 2018;57(12):1571-80.

18. Baekdal TA, Thomsen M, Kupčová V, Hansen CW, Anderson TW. Pharmacokinetics, safety, and tolerability of oral semaglutide in subjects with hepatic impairment. J Clin Pharmacol. 2018;58:1314-23.

19. Baekdal TA, Breitschaft A, Navarria A, Hansen CW. A randomized study investigating the effect of omeprazole on the pharmacokinetics of oral semaglutide. Expert Opin Drug Metab Toxicol. 2018;14(8):869-77.

20. Maarbjerg SJ, Borregaard J, Breitschaft A, Donsmark M, Søndergaard F. Evaluation of the effect of food on the pharmacokinetics of oral semaglutide. Diabetologia. 2017;60(Suppl 1):S69.

21. Meier J, Granhall C, Hoevelmann U, Navarria A, Plum-Moerschel $\mathrm{L}$, Ramesh C, et al. Efect of upper gastrointestinal disease on the pharmacokinetics of oral semaglutide in subjects with type 2 diabetes. Diabetes. 2019;68(Supp11):1013-P (Presented at the 79th Scientific Sessions of the American Diabetes Association Congress; 7-11 Jun 2019; San Francisco (CA)).

22. Hellriegel ET, Bjornsson TD, Hauck WW. Interpatient variability in bioavailability is related to the extent of absorption: implications for bioavailability and bioequivalence studies. Clin Pharmacol Ther. 1996;60(6):601-7.

23. Yamada Y, Katagiri H, Hamamoto Y, et al. Dose-response, efficacy, and safety of oral semaglutide monotherapy in Japanese patients with type 2 diabetes (PIONEER 9): a 52-week, phase 
2/3a, randomised, controlled trial. Lancet Diabetes Endocrinol. 2020;8(5):377-91.

24. Petri KCC, Ingwersen SH, Flint A, Zacho J, Overgaard RV. Exposure-response analysis for evaluation of semaglutide dose levels in type 2 diabetes. Diabetes Obes Metab. 2018;20:2238-45.

25. Graaf CD, Donnelly D, Wootten D, Lau J, Sexton PM, Miller LJ, et al. Glucagon-like peptide-1 and its class B G protein-coupled receptors: a long march to therapeutic successes. Pharmacol Rev. 2016;68(4):954-1013.

26. Mahato RI, Narang AS, Thoma L, Miller DD. Emerging trends in oral delivery of peptide and protein drugs. Crit Rev Ther Drug Carr Syst. 2003;20(2-3):153-214.
27. Granhall C, Donsmark M, Blicher TM, Golor G, Søndergaard FL, Thomsen M, et al. Safety and pharmacokinetics of single and multiple ascending doses of the novel oral human GLP-1 analogue, oral semaglutide, in healthy subjects and subjects with type 2 diabetes. Clin Pharmacokinet. 2019;58(6):781-91.

28. Hauge C, Breitschaft A, Hartoft-Nielsen M-L, Jensen S, Baekdal TA. A drug-drug interaction trial of oral semaglutide with levothyroxine and multiple coadministered tablets. J Endocrine Soc. 2019;3(Suppl 1):SAT-104. https://doi.org/10.1210/js. 2019-SAT-140. 